\title{
Flexible and reconfigurable radio frequency electronics realized by high-throughput screen printing of vanadium dioxide switches
}

\author{
Weiwei Li [1]', Mohammad Vaseem [1', Shuai Yang ${ }^{1}$ and Atif Shamim (1)
}

\begin{abstract}
Smart materials that can change their properties based on an applied stimulus are in high demand due to their suitability for reconfigurable electronics, such as tunable filters or antennas. In particular, materials that undergo a metal-insulator transition (MIT), for example, vanadium dioxide $\left(\mathrm{VO}_{2}\right)(\mathrm{M})$, are highly attractive due to their tunable electrical and optical properties at a low transition temperature of $68^{\circ} \mathrm{C}$. Although deposition of this material on a limited scale has been demonstrated through vacuum-based fabrication methods, its scalable application for largearea and high-volume processes is still challenging. Screen printing can be a viable option because of its highthroughput fabrication process on flexible substrates. In this work, we synthesize high-purity $\mathrm{VO}_{2}(\mathrm{M})$ microparticles and develop a screen-printable $\mathrm{VO}_{2}$ ink, enabling the large-area and high-resolution printing of $\mathrm{VO}_{2}$ switches on various substrates. The electrical properties of screen-printed $\mathrm{VO}_{2}$ switches at the microscale are thoroughly investigated under both thermal and electrical stimuli, and the switches exhibit a low ON resistance of 1.8 ohms and an ON/OFF ratio of more than 300. The electrical performance of the printed switches does not degrade even after multiple bending cycles and for bending radii as small as $1 \mathrm{~mm}$. As a proof of concept, a fully printed and mechanically flexible band-pass filter is demonstrated that utilizes these printed switches as reconfigurable elements. Based on the $\mathrm{ON}$ and OFF conditions of the $\mathrm{VO}_{2}$ switches, the filter can reconfigure its operating frequency from 3.95 to $3.77 \mathrm{GHz}$ without any degradation in performance during bending.
\end{abstract}

\section{Introduction}

In recent years, smart electronics have gained tremendous attention in both scientific and industrial fields ${ }^{1}$ due to their ability to transform, change their shape or tuning, and modulate their properties in response to external stimuli, such as mechanical deformation ${ }^{2}$, thermal heating ${ }^{3}$, an electrical field ${ }^{4}$, or a magnetic force ${ }^{5}$. Creating smart electronics and exploring their versatile applications in smart energy devices ${ }^{4,6}$, smart skins ${ }^{7,8}$, smart wearables $^{9-11}$, reconfigurable electronics ${ }^{12-14}$, and even smart cities $^{15}$, has raised new requirements for

\footnotetext{
Correspondence: Weiwei Li (weiwei.li@kaust.edu.sa)

'IMPACT Lab, Computer, Electrical and Mathematical Sciences and Engineering (CEMSE) Divisiorn, King Abdullah University of Science and Technology (KAUST), Thuwal 23955-6900, Kingdom of Saudi Arabia

These authors contributed equally: Weiwei Li, Mohammad Vaseem
}

functional materials that can tune their properties according to specific demands ${ }^{6,13,16,17}$. Despite a diverse cluster of new materials, such as carbon-based materials $^{2,10}$, transition metal dichalcogenides ${ }^{18}$, and metal oxide nanocrystals ${ }^{19}$, metal insulator transition (MIT) materials have great promise due to their easy and reversible tunability between the metal and insulator via various external stimuli ${ }^{20-23}$. For example, vanadium dioxide $\left(\mathrm{VO}_{2}\right)(\mathrm{M})$ exhibits MIT behavior by possessing an insulating state at room temperature and a metallic state at a critical temperature $\left(\sim 68^{\circ} \mathrm{C}\right)^{24}$ and is attracting a substantial amount of interest for optical and electrical electronic applications ${ }^{12,17,25-29}$. Currently, a variety of techniques, such as radio frequency (RF) sputtering ${ }^{30,31}$, pulsed laser deposition ${ }^{32}$, and electron beam evaporation $^{29}$, have been evaluated to fabricate high-quality $\mathrm{VO}_{2}$

\section{(c) The Author(s) 2020}

(c) (i) Open Access This article is licensed under a Creative Commons Attribution 4.0 International License, which permits use, sharing, adaptation, distribution and reproduction cc in any medium or format, as long as you give appropriate credit to the original author(s) and the source, provide a link to the Creative Commons license, and indicate if changes were made. The images or other third party material in this article are included in the article's Creative Commons license, unless indicated otherwise in a credit line to the material. If material is not included in the article's Creative Commons license and your intended use is not permitted by statutory regulation or exceeds the permitted use, you will need to obtain permission directly from the copyright holder. To view a copy of this license, visit http://creativecommons.org/licenses/by/4.0/. 
films with an ON/OFF ratio of over $10^{4}$. Unfortunately, these previously reported deposition methods operate under high vacuum conditions. They either consist of complex steps or deposit $\mathrm{VO}_{2}$ thin films with a limited size, which is not favorable for efficient fabrication. In addition, a high processing temperature is typically required during deposition, limiting compatibility with flexible polymer substrates.

Recently, printed electronics have made rapid progress. By depositing functional materials via conventional printing techniques (e.g., inkjet printing and screen printing), printed electronics offer a new method for achieving highthroughput and roll-to-roll production of electronic devices and integrated systems at low $\operatorname{cost}^{33-38}$. Despite the great progress made in printable materials and printing technologies, few reports have succeeded in developing printable $\mathrm{VO}_{2}$ ink to deposit high-quality $\mathrm{VO}_{2}$ films. Ji et al. prepared an inkjet-printable $\mathrm{VO}_{2}$ ink, fabricated $\mathrm{VO}_{2}$ films, and studied the infrared thermochromic properties ${ }^{39}$. Our group reported a $\mathrm{VO}_{2}$ nanoparticle-based ink for the inkjet printing of $\mathrm{VO}_{2}$ films. The printed $\mathrm{VO}_{2}$ films demonstrated an electrical conductivity of $\sim 1 \mathrm{~S} \mathrm{~m}^{-1}$ in the insulating state and $150-200 \mathrm{~S} \mathrm{~m}^{-1}$ in the metallic state, resulting in a conductivity ratio of $10^{217}$. The ink has also been used to fabricate RF switches, exhibiting decent RF performance from low frequencies $(10 \mathrm{MHz})$ up to $40 \mathrm{GHz}^{12}$, which is comparable to that of nonprinted $\mathrm{VO}_{2}$ switches $^{29}$. However, these inkjet printable $\mathrm{VO}_{2}$ inks suffer from low $\mathrm{VO}_{2}$ loading, possible nozzle clogging, unstable printing, and slow deposition. The printed $\mathrm{VO}_{2}$ films exhibit poor adhesion to substrates and less flexibility due to the absence of polymer additives. In addition, to achieve improved electrical performance, thick films (tens of micrometers) are typically required; thus, tens or hundreds of printing passes are necessary to generate high-quality $\mathrm{VO}_{2}$ films, which is not favorable for high-efficiency fabrication, especially for largearea printing.

In this work, we synthesize high-crystal $\mathrm{VO}_{2}$ (M) microparticles and report a simple route to produce $\mathrm{VO}_{2}$ ink suitable for screen-printing techniques. With this ink, we demonstrate high-throughput printing of $\mathrm{VO}_{2}$ switches on both flexible polymers (Kapton and poly [ethylene terephthalate] (PET)) and rigid sapphire wafers with high resolution (down to $60 \mu \mathrm{m}$ ) at high speed $\left(220 \mathrm{~mm} \mathrm{~s}^{-1}\right)$ over a large area. The printed $\mathrm{VO}_{2}$ switches exhibit excellent electrical performance (ON/OFF ratio of 300 and conductivity as high as $1037 \mathrm{~S} \mathrm{~m}^{-1}$ after heating at $120^{\circ} \mathrm{C}$ ), excellent mechanical stability (bending radius down to $1 \mathrm{~mm}$ ), satisfactory air stability (negligible performance change after 1 month), and decent RF performance $(-1 \mathrm{~dB}$ isolation and $-2.6 \mathrm{~dB}$ insertion loss up to $20 \mathrm{GHz}$ ). Finally, we demonstrate fully screen-printed reconfigurable RF electronic devices, including series switches, band-stop filters, and band-pass filters.

\section{Results}

In our previous study ${ }^{17}$, we determined that as-prepared $\mathrm{VO}_{2}(\mathrm{M})$ nanoparticles do not have high quality due to a low crystallinity, which resulted in poor electrical performance. In this work, we prepared highly crystalline $\mathrm{VO}_{2}$ microparticles using an autoclave reactor in an aqueous environment using a commercially available $\mathrm{V}_{2} \mathrm{O}_{5}$ powder ( $\geq 98 \%$, Sigma-Aldrich) as the precursor and oxalic acid as the reducing agent. Initially, $\mathrm{X}$-ray diffraction (XRD) analysis and morphological observations were conducted for $\mathrm{V}_{2} \mathrm{O}_{5}$ powder, as presented in Fig. S1. It is clearly shown that the $\mathrm{V}_{2} \mathrm{O}_{5}$ powder consisted of micronsized particles, and multiple crystalline peaks were observed in the XRD pattern. We designed experiments using a constant weight percentage of precursor materials with different reaction times (i.e., 3,6 , and $24 \mathrm{~h}$ ), and the products were investigated by XRD analysis. As displayed in Fig. S2, the as-synthesized samples were a mixture of undesired $\mathrm{VO}_{2}(\mathrm{~A})$ and desired $\mathrm{VO}_{2}(\mathrm{M})$ phases that produced low-intensity XRD peaks. To achieve a pure $\mathrm{VO}_{2}(\mathrm{M})$ phase, we exposed the as-synthesized samples to different annealing environments. The XRD spectra demonstrated in Fig. 1a clearly reveal that a pure $\mathrm{VO}_{2}(\mathrm{M})$ phase was obtained for all the samples synthesized for 3-, 6- and 24-h reaction times after annealing at $300^{\circ} \mathrm{C}$ in vacuum. The XRD peaks observed at $27.84^{\circ}, 37.01^{\circ}$, $42.19^{\circ}$, and $55.56^{\circ}$ were indexed to the [011], [200], [210] and [-222] crystal planes, which is consistent with JCPDS No. 72-0514. It is interesting to note that the sample synthesized for $3 \mathrm{~h}$ followed by annealing demonstrated low-intensity XRD peaks, while the 6- and 24-h reaction time samples demonstrated higher intensity peaks. Thus, $\mathrm{VO}_{2}$ particles synthesized with a reaction time of $6 \mathrm{~h}$ and an annealing temperature of $300^{\circ} \mathrm{C}$ were used for further study. It is noteworthy that the reaction time is much shorter than those reported in the literature, where more than one day has been used for the synthesis ${ }^{40-42}$.

The as-annealed products were further characterized for their morphological and thermal behavior. It can be seen in the scanning electron microscopy (SEM) image in Fig. $1 \mathrm{~b}$ that the $\mathrm{VO}_{2}$ particles were uniformly grown and comprised micron-scale sheets that were arranged in a flower-shaped morphology, with an average particle size from 4-6 $\mu \mathrm{m}$ (inset in Fig. 1b). It is interesting to note that the morphology of the as-annealed products is similar to that of the $\mathrm{V}_{2} \mathrm{O}_{5}$ precursor powder. Thus, the shape and size of the $\mathrm{VO}_{2}$ particles can be further optimized by selecting the finest $\mathrm{V}_{2} \mathrm{O}_{5}$ powder with regular sizes either from commercially available sources or through custom in-house preparation of $\mathrm{V}_{2} \mathrm{O}_{5}$ particles. A micron-thick sheet was also characterized by transmission electron microscopy (TEM), as illustrated in Fig. 1c, d. The inset in Fig. 1c displays a selected-area electron diffraction (SAED) pattern of the sheet, which confirmed its 


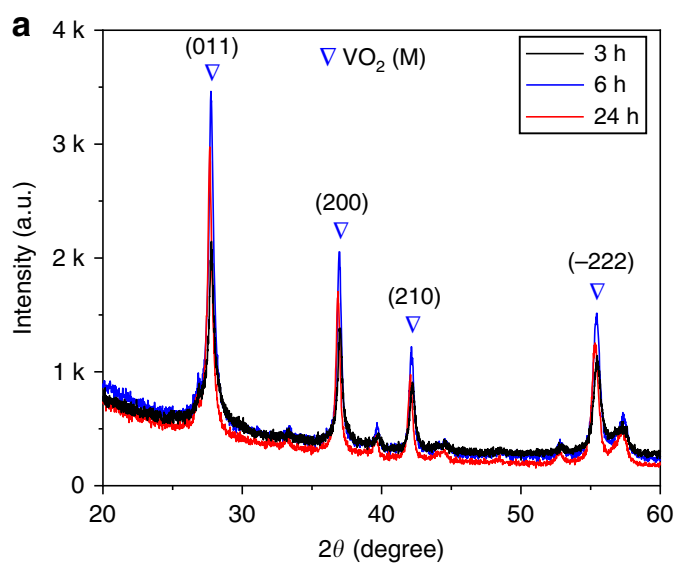

C

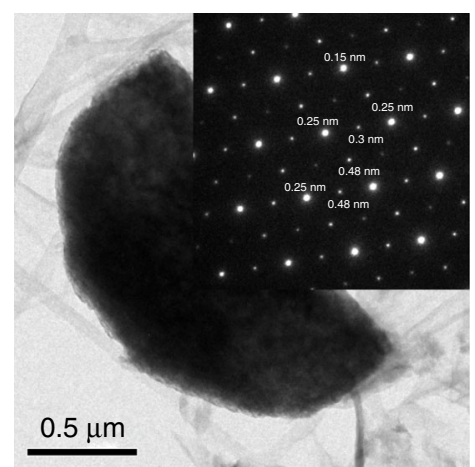

d

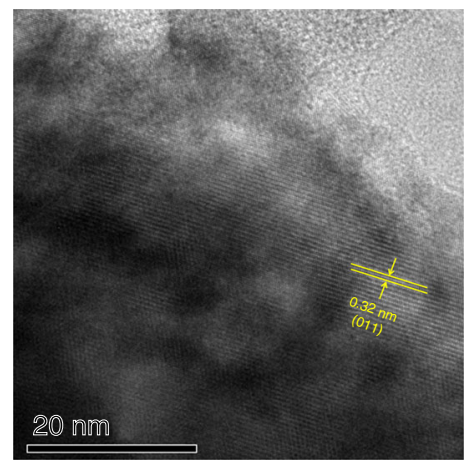

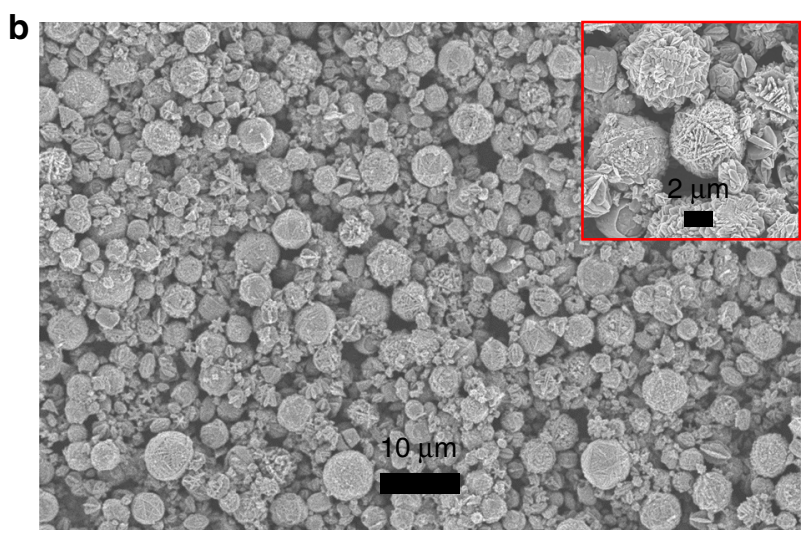

e

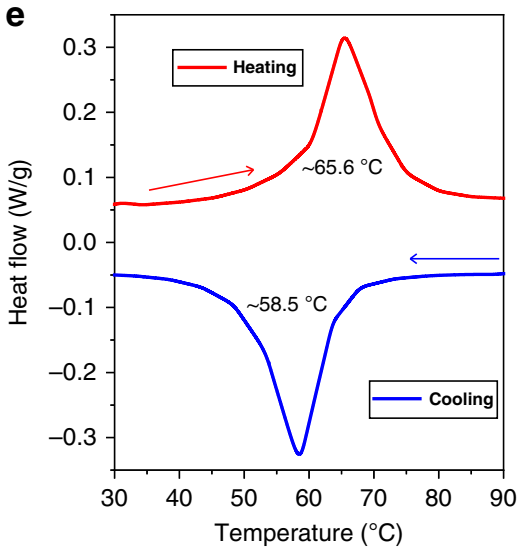

Fig. 1 Characterization of the $\mathrm{VO}_{2}$ microparticles after annealing at $300^{\circ} \mathrm{C}$ for $\mathbf{3} \mathbf{h}$ in vacuum. a XRD spectra for the different reaction times, b SEM images, c TEM image and the corresponding SAED pattern, d HR-TEM image, and e DSC spectrum

crystalline nature with interplanar distances of $0.15,0.25$, 0.3 , and $0.48 \mathrm{~nm}$. The high-resolution TEM (HR-TEM) image displayed in Fig. 1d indicates that the distance between two crystal planes was $0.32 \mathrm{~nm}$, which corresponds to (011) crystal planes. The existence of an MIT temperature is an attractive property of $\mathrm{VO}_{2}$, and it was assessed via thermal analysis using differential scanning calorimetry (DSC), as displayed in Fig. 1e. The annealed $\mathrm{VO}_{2}$ particles were exposed to heating and cooling from room temperature to $90^{\circ} \mathrm{C}$. Two clear MIT peaks at $\sim 65.6^{\circ} \mathrm{C}$ during heating and $\sim 58.5^{\circ} \mathrm{C}$ during cooling are observed. The calculated thermal width was $\sim 7^{\circ} \mathrm{C}$, which corresponds to a first-order phase transition. The thermal width is lower than that of previously reported $\mathrm{VO}_{2}$ nanoparticles $\left(\sim 18^{\circ} \mathrm{C}\right)^{17}$ because of the high quality of the as-annealed $\mathrm{VO}_{2}$ particles, which is of importance in smart optical and electrical switches ${ }^{43}$. It should be noted that the MIT temperature is an inherent property of the $\mathrm{VO}_{2}(\mathrm{M})$ phase, and its bulk value is $\sim 68^{\circ} \mathrm{C}$. In our case, we observed that the MIT temperature of the as-annealed $\mathrm{VO}_{2}$ particles is slightly lower than that of the bulk VO2 particles, which is most likely due to the impurity of the precursor (i.e., $\mathrm{V}_{2} \mathrm{O}_{5}, \geq 98 \%$ ) used for the synthesis. It is well known that impurities and doping have prominent effects on the MIT temperature, and even 1-2\% impurities/doping can decrease the MIT temperature ${ }^{44-46}$. We observed that the MIT temperature of the as-synthesized $\mathrm{VO}_{2}$ particles (without annealing) was not distinct because the phase was not in a pure state, as confirmed by the DSC spectrum in Fig. S3a. Once the pure $\mathrm{VO}_{2}(\mathrm{M})$ phase was achieved after annealing, similar MIT temperatures were observed. This was confirmed by the sample with a long reaction time of $24 \mathrm{~h}$, where an MIT temperature of $65.69^{\circ} \mathrm{C}$ was obtained (Fig. S3b).

It is clear from the results in Fig. 1 that pure $\mathrm{VO}_{2}(\mathrm{M})$ particles were obtained. To develop a screen-printable $\mathrm{VO}_{2}$ ink, a mixed solvent of terpineol and ethanol (Fig. 2a) was selected due to its high viscosity and low surface tension. Ethyl cellulose (EC) (Fig. 2a) was used as an organic binder, dispersing agent, and rheological modifier. In a typical ink formulation, EC was first mixed with terpineol and ethanol at a weight ratio of 1:4:0.4 to form a viscous solution. Then, the $\mathrm{VO}_{2}$ particles were mixed with the prepared solution at a weight ratio of $3: 5$, followed by 

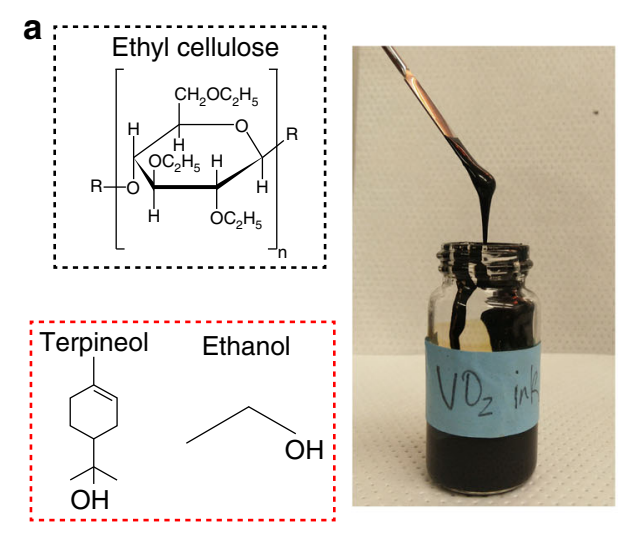
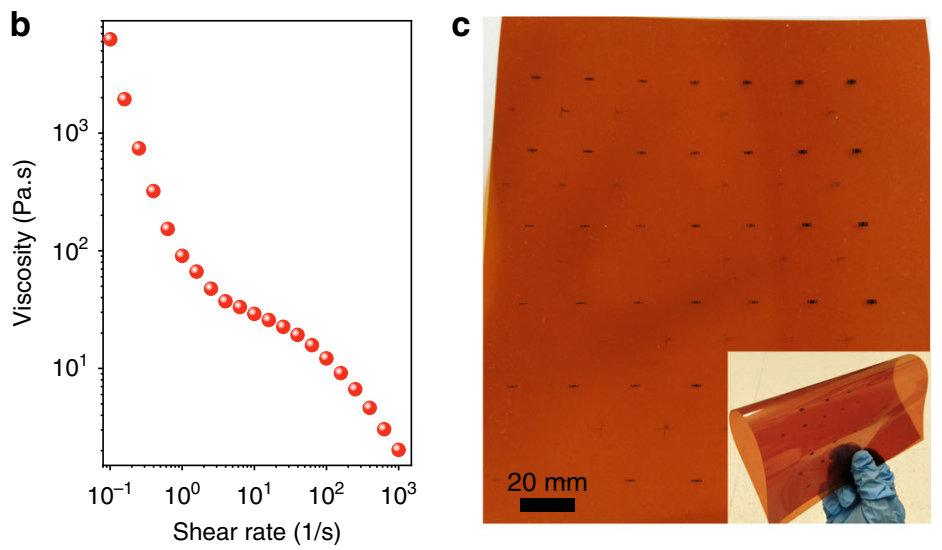

d

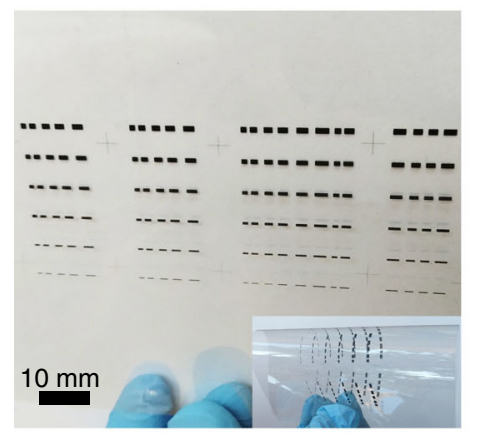

e

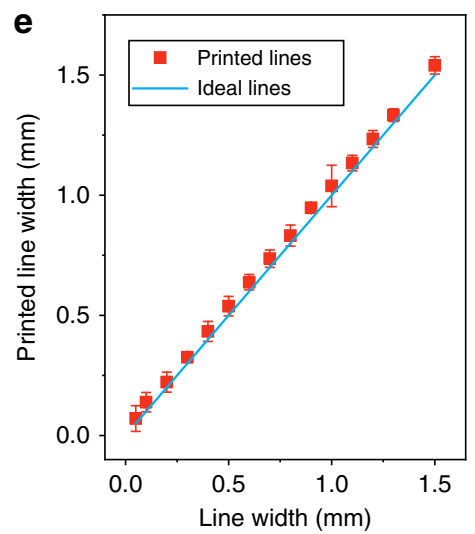

f

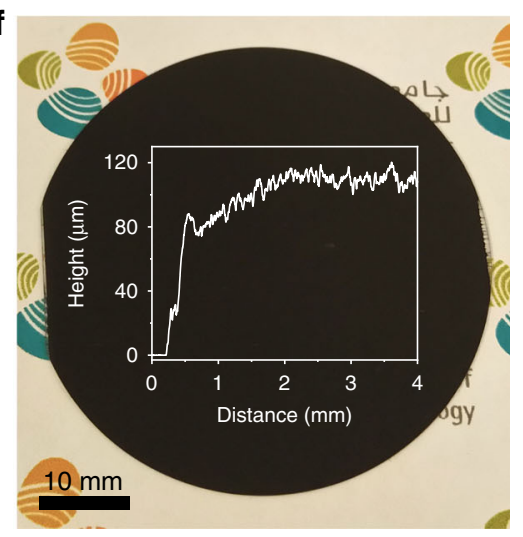

Fig. $2 \mathrm{VO}_{2}$ ink properties and large-area printing of $\mathrm{VO}_{2}$ switches. a Molecular structures of binder (ethyl cellulose) and solvents (terpineol and ethanol) and digital photograph of the prepared $\mathrm{VO}_{2}$ ink. $\mathbf{b}$ The measured viscosity of the $\mathrm{VO}_{2}$ ink at a shear rate from 0.1 to $1000 \mathrm{~s}^{-1}$. $\mathbf{c}$ Digital photographs of the screen-printed $\mathrm{VO}_{2}$ switches on a Kapton substrate. Inset: Bent $\mathrm{VO}_{2}$ switches. $\mathbf{d}$ Digital photographs of the screen-printed $\mathrm{VO}_{2}$ switches on a PET substrate. Inset: Bent $\mathrm{VO}_{2}$ switches. e The printed $\mathrm{VO}_{2}$ line width as a function of the designed line width on the screen mesh. f Digital photograph of the screen-printed $\mathrm{VO}_{2}$ film on a 2-inch sapphire wafer. Inset: profilometry data from the printed $\mathrm{VO}_{2}$ layer

agitation to obtain a homogenous and stable $\mathrm{VO}_{2}$ ink with a $\mathrm{VO}_{2}$ content of 37.5 wt.\% (Fig. 2a). The viscosity of the resultant ink was measured using a rheometer at a shear rate from 0.1 to $1000 \mathrm{~s}^{-1}$, and the curve is displayed in Fig. $2 \mathrm{~b}$. We clearly observed a shear thinning behavior of the formulated $\mathrm{VO}_{2}$ ink. The viscosity decreased from 12,492 to $58 \mathrm{~Pa} \mathrm{~s}$ as the shear rate increased from 0.1 to $10 \mathrm{~s}^{-1}$. This type of fluid behavior and high viscosity are essential to provide favorable printability. This ink was directly used for screen printing to deposit $\mathrm{VO}_{2}$ switches on various substrates. What should be noted is that the single-pass thickness of the printed $\mathrm{VO}_{2}$ film was affected by many factors, such as the screen mesh parameters (i.e., mesh count, mesh diameter, and emulsion thickness), printing parameters (i.e., printing speed, squeegee pressure, and snap-off distance), and ink properties (i.e., solid content and viscosity $)^{47,48}$. Typically, a smaller mesh count, thinner wire, thicker emulsion, slower speed, larger pressure, larger snap-off distance, more solid content, and higher viscosity print thicker films. However, among all these factors, the mesh count and the solid content (i.e., $\mathrm{VO}_{2}$ particles and EC) of the ink are critical for controlling the thickness of the printed films. Specifically, a smaller mesh count means fewer openings per inch and a larger size of each opening in the screen, which results in a thicker film. In our experiment, the mesh count was fixed as 325. Thus, the dominant factor controlling the single-pass thickness was the solid content. A low solid content results in a thin layer. However, this might produce additional pores in the film due to the high amount of solvent. As a result, the electrical performance (i.e., ON/OFF ratio) of the printed $\mathrm{VO}_{2}$ films worsened, as shown in Fig. S4. After considering the optimized ink formulation (i.e., solid content and viscosity) and the printing parameters (i.e., printing speed and mesh count), the resultant thickness of the printed $\mathrm{VO}_{2}$ film in a single pass was $\sim 8-10 \mu \mathrm{m}$. After the screen-printing process, the printed $\mathrm{VO}_{2}$ switches were baked in an oven at $120{ }^{\circ} \mathrm{C}$ for $1 \mathrm{~h}$ to evaporate the solvents and sinter the $\mathrm{VO}_{2}$ film. It is noteworthy that the printed $\mathrm{VO}_{2}$ films without polymer 
binders contained particle aggregates and cracks after thermal sintering, as can be observed from the optical images in Fig. S5. The limited addition of the polymer binder (the weight ratio of $\mathrm{VO}_{2}$ to binder is $\sim 3.3$ ) was favorable for dispersing the $\mathrm{VO}_{2}$ particles and achieving flexibility of the printed $\mathrm{VO}_{2}$ film.

The low processing temperature enabled our $\mathrm{VO}_{2}$ ink to be suitable for various substrates, such as flexible polymers or even paper, largely widening its applications. Fig. 2c, d display digital photographs of the large-area $\mathrm{VO}_{2}$ switches printed on flexible Kapton and PET substrates in flat and bent states, respectively. We investigated the printed $\mathrm{VO}_{2}$ line features by comparing the printed line widths with the designed lines on the screen mesh. As presented in Fig. 2e, a linear behavior was observed between the printed and designed lines over the whole line width range (from $50 \mu \mathrm{m}$ to $1.5 \mathrm{~mm}$ ), demonstrating the excellent reliability of the screen-printed $\mathrm{VO}_{2}$ switches. The narrowest line width was $\sim 65 \mu \mathrm{m}$. In addition, we found that the printed $\mathrm{VO}_{2}$ lines were slightly wider $(\sim 30 \mu \mathrm{m})$ than the designed lines, which was attributed to ink spreading after transfer to the substrates. We further printed a thick and large $\mathrm{VO}_{2}$ film on a 2-inch sapphire wafer with 15 printing passes to demonstrate high-throughput printing, as displayed in Fig. 2f. The printed $\mathrm{VO}_{2}$ film had a thickness of $\sim 120 \mu \mathrm{m}$, as identified by the profile curve (inset in Fig. 2f), which was obtained by scanning the film with a profilometer (from the edge to the center). From the curve, we can also observe that the surface of the printed $\mathrm{VO}_{2}$ film was not smooth, which was attributed to the micron-sized particles. Notably, the printed wafer-scale $\mathrm{VO}_{2}$ film was of high quality, as confirmed by XRD pattern (Fig. S6), where four distinct diffraction peaks were indexed to pure $\mathrm{VO}_{2}(\mathrm{M})$ without any impurities. It should be noted that this film was printed at a high speed of $220 \mathrm{~mm} \mathrm{~s}^{-1}$, and it took only a few minutes to print the $\mathrm{VO}_{2}$ film. In comparison, tens of hours or even days are necessary to deposit the same $\mathrm{VO}_{2}$ film using the inkjet printing technique. All these results imply that our developed screen-printable $\mathrm{VO}_{2}$ ink and the conducted screen-printing process are ideal for mass production of $\mathrm{VO}_{2}$-based switches for tunable and reconfigurable RF electronics.

We then evaluated the electrical properties of the screen-printed $\mathrm{VO}_{2}$ switches. Figure 3a presents the apparatus for the current-voltage (I-V) measurements, in which two probe tips were connected to the samples and a source measurement unit to measure the resistance of the $\mathrm{VO}_{2}$ switches. $\mathrm{VO}_{2}$ was printed between two silver (Ag) electrodes. The electrode width and the gap between the electrodes were marked as the width and length of the $\mathrm{VO}_{2}$ switches, respectively. Figure $3 \mathrm{~b}$ displays the measured resistance of the printed $\mathrm{VO}_{2}$ switches (width and length were 0.5 and $0.3 \mathrm{~mm}$, respectively) with increasing printing passes from one to nine at $25^{\circ} \mathrm{C}$ (called the OFF state) and $90^{\circ} \mathrm{C}$ (called the $\mathrm{ON}$ state). The resistance at both the OFF and ON states decreased rapidly for a larger printing pass, as a thicker and denser $\mathrm{VO}_{2}$ layer was formed. Specifically, the OFF resistance was $1976 \mathrm{ohm}$, while the $\mathrm{ON}$ resistance was $7.3 \mathrm{ohms}$ after seven printing passes, yielding an ON/OFF ratio of 270, which is much higher than that of inkjet-printed $\mathrm{VO}_{2}$ films ${ }^{12,17}$. The reason for this was attributed to the (1) high quality of the $\mathrm{VO}_{2}$ particles, as confirmed by the intense XRD peaks; (2) well-dispersed $\mathrm{VO}_{2}$ particles in the ink system; and (3) dense $\mathrm{VO}_{2}$ layer via polymer binding. We then investigated the resistance of the $\mathrm{VO}_{2}$ switches with various lengths. As presented in Fig. 3c, when the $\mathrm{VO}_{2}$ film width was fixed as $0.5 \mathrm{~mm}$, the measured $\mathrm{ON}$ resistance linearly increased from 1.8 to $10.3 \mathrm{ohms}$ as the $\mathrm{VO}_{2}$ length increased from 0.035 to $0.4 \mathrm{~mm}$, respectively. Thus, $\mathrm{VO}_{2}$ switches with varying $\mathrm{ON}$ and OFF resistances (within the bound of the best-case scenarios) can be designed by selecting different geometric parameters of the $\mathrm{VO}_{2}$ film, such as length, width, and thickness. The electrical conductivity $(\sigma)$ of the printed $\mathrm{VO}_{2}$ switches in the ON state was calculated based on the measured $\mathrm{ON}$ resistance $(R)$ and the $\mathrm{VO}_{2}$ geometries, such as $\mathrm{VO}_{2}$ length $(l), \mathrm{VO}_{2}$ width $(w)$, and $\mathrm{VO}_{2}$ thickness $(t)$, through the following equation, $\sigma=l /(R w t)$. The measured thicknesses and the calculated conductivities are displayed in Fig. S7 and Fig. 3d, respectively. Various samples for each length were measured, and an average conductivity value was obtained for each length. Generally, the average conductivity values in the ON state for all the samples are almost similar with some minor fluctuations (in the range of 852 to $1037 \mathrm{~S}$ $\mathrm{m}^{-1}$ ). These values are superior to the value of $200 \mathrm{~S} \mathrm{~m}^{-1}$ obtained for inkjet-printed $\mathrm{VO}_{2}$ films ${ }^{12}$. The slight fluctuation in the calculated conductivity can be attributed to fabrication tolerances because the samples with different lengths had slightly different thicknesses and widths. Furthermore, the edges of printed films are typically not smooth, and the surfaces are relatively rough, which can further affect the calculated results. Nevertheless, such a high ON/OFF ratio and electrical conductivity are promising for printed $\mathrm{VO}_{2}$ films that have been reported. Note that the achieved ON/OFF ratio $\left(\sim 10^{2}\right)$ is low compared to the typical values achieved from the $\mathrm{VO}_{2}$ films deposited through nanofabrication techniques $\left(\sim 10^{4}\right)$ due to the irregular $\mathrm{VO}_{2}$ particles during synthesis, partially covering of $\mathrm{VO}_{2}$ particles by polymer binders (Fig. S8), and the pores in the printed films. However, printed films have the advantages of easy processing, direct patterning, and roll-to-roll production capability. With the advancement of printing techniques and inks, it is expected that in the near future, printed films will have a comparable performance to films deposited through nanofabrication techniques. 

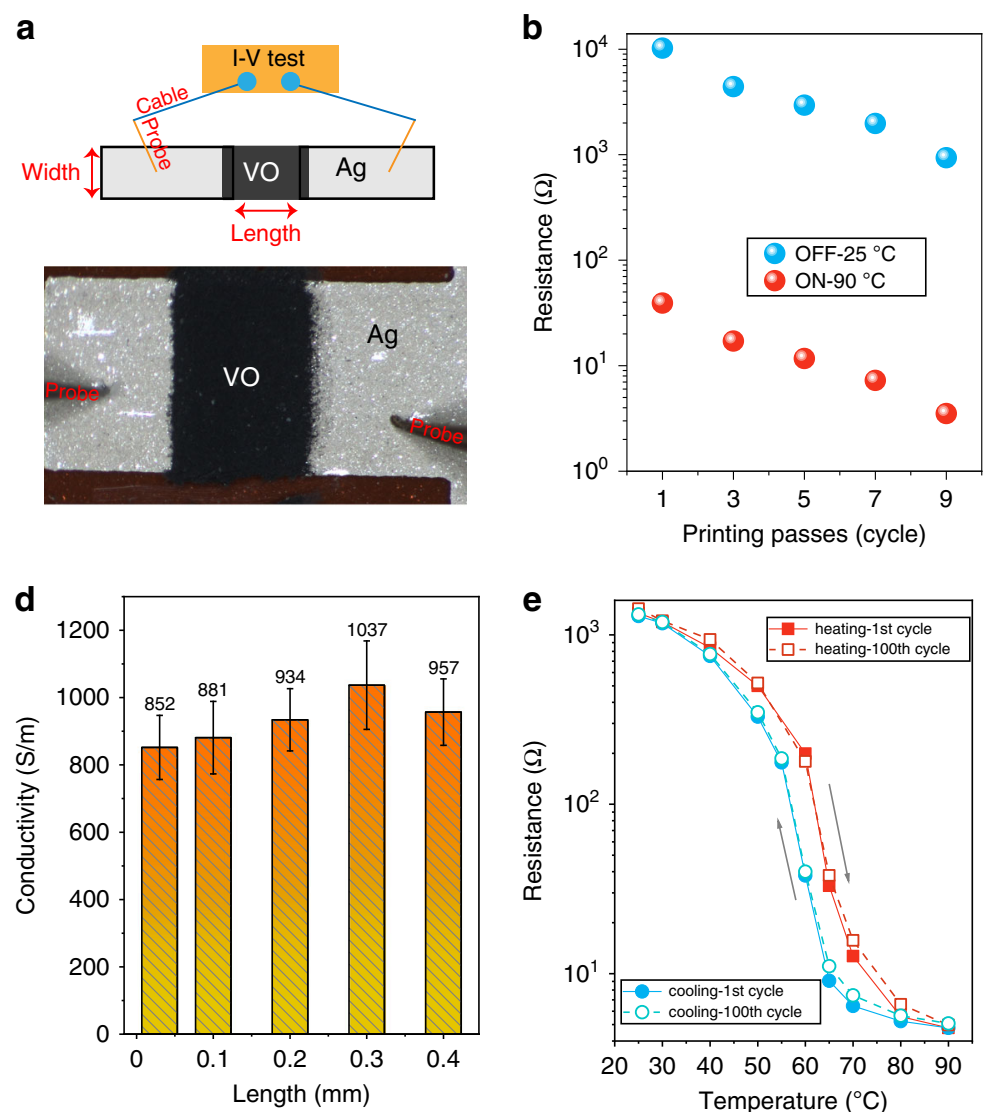
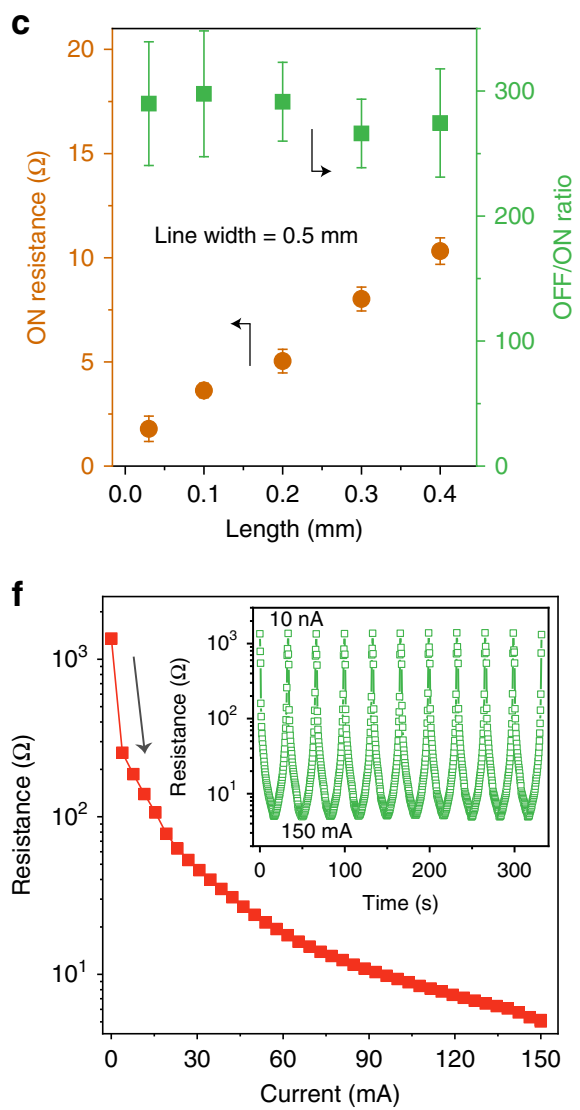

Fig. 3 Electrical performance of the printed $\mathbf{V O}_{2}$ switches. a The setup diagram (top panel) to measure the resistance of the printed $\mathrm{VO} \mathrm{O}_{2}$ switch Bottom panel: $\mathrm{VO}_{2}$ switch under test. b The measured resistance of the $\mathrm{VO}_{2}$ switches as a function of printing passes at $25^{\circ} \mathrm{C}\left(\mathrm{OFF}\right.$ state) and $90{ }^{\circ} \mathrm{C}$ (ON state). c The measured ON resistance and ON/OFF ratio of the $\mathrm{VO}_{2}$ switches as a function of switch length. $\mathbf{d}$ The calculated electrical conductivity of the printed $\mathrm{VO}_{2}$ switches as a function of switch length. e The measured resistance of the printed $\mathrm{VO}_{2}$ switch at different temperatures in the heating and cooling process. $\mathbf{f}$ The measured resistance of the printed $\mathrm{VO}_{2}$ switch at different bias currents of $10 \mathrm{nA}$ and $150 \mathrm{~mA}$. Inset: the resistance as a function of cyclic electrical triggering

As a material with an MIT, $\mathrm{VO}_{2}$ could be stimulated by various external stimuli. Here, we evaluated the electrical response of the printed $\mathrm{VO}_{2}$ switches by applying heat and a bias current. We selected a typical $\mathrm{VO}_{2}$ switch for further study that had a length of $0.2 \mathrm{~mm}$, a width of $0.5 \mathrm{~mm}$, and an $\mathrm{ON}$ resistance of $\sim 5 \mathrm{ohms}$. As displayed in Fig. 3e, at room temperature $\left(25^{\circ} \mathrm{C}\right)$, the measured resistance was $\sim 1300 \mathrm{ohms}$, exhibiting insulating behavior. Then, the resistance slowly decreased to $200 \mathrm{ohms}$ as the temperature increased to $60{ }^{\circ} \mathrm{C}$. When the temperature reached $65^{\circ} \mathrm{C}$, the resistance dropped dramatically to 33 ohms, exhibiting an $83.5 \%$ decrease compared with the resistance at $60^{\circ} \mathrm{C}$. This is consistent with the transition temperature of $65.6^{\circ} \mathrm{C}$ observed in the DSC result (Fig. 1e). With a further increase in the temperature, the resistance decreased to $5.5 \mathrm{ohms}$ at $80{ }^{\circ} \mathrm{C}$ and remained constant at a value of approximately $5 \mathrm{ohms}$ at $90^{\circ} \mathrm{C}$. During the cooling process, the resistance increased from $5 \mathrm{ohms}$ to $\sim 1300$ ohms when the temperature decreased from $90^{\circ} \mathrm{C}$ to $25^{\circ} \mathrm{C}$, respectively. Notably, this resistance change with temperature was highly repeatable, as the hundredth heating and cooling of the same sample demonstrated a similar electrical response in both the trend for the resistance change and the OFF and ON resistances, despite a slight variation in some of the resistance values. A similar resistance response was observed by applying different bias currents to the $\mathrm{VO}_{2}$ switches, as presented in Fig. 3f. The initial resistance at an applied current of $10 \mathrm{nA}$ was $1351 \mathrm{ohms}$, which was in the OFF state. The resistance dropped dramatically to 254 ohms at a supplying current of $3.6 \mathrm{~mA}$ and finally reduced to $5.1 \mathrm{ohms}$ when the current was $150 \mathrm{~mA}$. As the current further increased to $160 \mathrm{~mA}$, the $\mathrm{VO}_{2}$ switch was burned, implying that careful selection of the applied current is important and that the current should be increased step by step. Similar to heat triggering, the resistance response of the $\mathrm{VO}_{2}$ switches with electrical triggering demonstrated excellent repeatability via the cyclic application of 

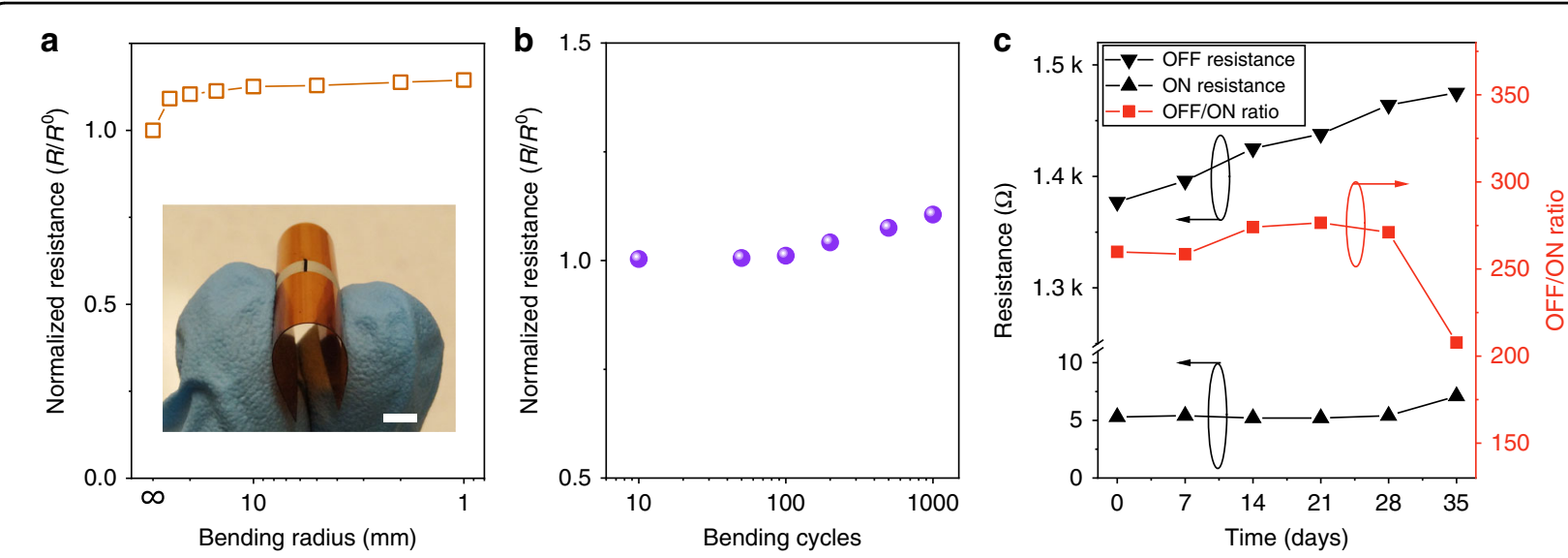

Fig. 4 The mechanical and air stability of the printed $\mathrm{VO}_{2}$ switches. a The normalized resistance of the $\mathrm{VO}_{2}$ switch under different bending radii. Inset: digital photograph of the $\mathrm{VO}_{2}$ switch under bending. The scale bar is $5 \mathrm{~mm}$. b The normalized resistance of the $\mathrm{VO}_{2}$ switch under cyclic bending with bending radii from infinite to $1 \mathrm{~mm}$. c The measured resistance and ON/OFF ratio of the $\mathrm{VO}_{2}$ switch at different times after environmental exposure

currents of $10 \mathrm{nA}$ and $150 \mathrm{~mA}$ (inset in Fig. 3f). What should be noted is that the geometric parameters of a $\mathrm{VO}_{2}$ switch, such as its length, width, and thickness, determine the biasing current applied to the switch. Typically, a wider, longer, and thicker $\mathrm{VO}_{2}$ switch requires a higher biasing current ${ }^{12,29}$.

The printed $\mathrm{VO}_{2}$ switches not only demonstrated a high ON/OFF ratio, electrical conductivity, and triggering repeatability but also presented excellent flexibility and mechanical stability. We evaluated the resistance change of a typical printed $\mathrm{VO}_{2}$ switch by applying various bending radii and repeated bending cycles. As presented in Fig. 4a, the resistance of the $\mathrm{VO}_{2}$ film with a bending radius of $25 \mathrm{~mm}$ increased by $10 \%$ compared with that in its flat state (infinite radii), which was attributed to tensile release. As the bending radii decreased, the resistance became relatively stable and exhibited negligible degradation ( 4\%), even when the radius was reduced to $1 \mathrm{~mm}$. In addition, the resistance was retained even under repeated bending of 1000 cycles with a bending radius from infinite to $1 \mathrm{~mm}$, though an $11 \%$ increase in resistance was observed after the bending test, as depicted in Fig. 4b. This excellent mechanical stability was attributed to the polymer binders between the $\mathrm{VO}_{2}$ particles, which largely enhanced the particle interconnections and provided a fixed network to diminish film cracks, enabling a reliable $\mathrm{VO}_{2}$ switch for practical use. In addition to the flexibility, we evaluated the long-term stability of the printed $\mathrm{VO}_{2}$ switches by examining the resistance changes in a typical indoor environment (temperature: $25^{\circ} \mathrm{C}$, humidity: 55\%). As depicted in Fig. 4c, the $\mathrm{VO}_{2}$ switches exhibited outstanding durability, in which the ON resistance was constant even after exposure to air without any protection for one month. The slightly increased ON/OFF ratio was due to an increase in the OFF resistance, which was attributed to the absorbed moisture on the surface of the $\mathrm{VO}_{2}$ switches. The OFF resistance recovered to its initial value after heating at $100^{\circ} \mathrm{C}$ for $10 \mathrm{~min}$. After one month, the $\mathrm{ON}$ resistance began to increase, which caused a sharp decrease in the ON/OFF ratio. Nevertheless, our printed $\mathrm{VO}_{2}$ switches demonstrated highly stable behavior under mechanical deformation and in air, which is essential and necessary for practical applications.

In addition to the electrical properties of the printed $\mathrm{VO}_{2}$ switches, the RF performance is another critical factor that was investigated for potential applications in reconfigurable RF electronics; specifically, a high RF isolation and transmission in the OFF and ON states, respectively, are important. We first printed a coplanar waveguide (CPW)-based series switch on a sapphire wafer using Ag ink (Fig. 5a). The series switch contained two ground lines separated by one signal line; a gap was left in the middle of the signal line to print the $\mathrm{VO}_{2}$ switch (width: $500 \mu \mathrm{m}$, length: $200 \mu \mathrm{m}$ ) (Fig. 5b) that acted as a switch to control the RF signal being transmitted between the two ends of the signal line. In the OFF state, the $\mathrm{VO}_{2}$ switches were deactivated, and thus, the resistance of the $\mathrm{VO}_{2}$ switch was high enough $(\sim 1500 \mathrm{ohm})$ to block the $\mathrm{RF}$ signal for transmission. In contrast, the RF signal could transmit in the signal line when the resistance of the $\mathrm{VO}_{2}$ switch was low and in the activated status $(\sim 5 \mathrm{ohm})$. The $\mathrm{RF}$ performance for the printed $\mathrm{VO}_{2}$ switches was examined by investigating the scattering parameters (Sparameters S11, S12, S21, and S21) of the series switch via a two-port measurement (Fig. 5b). Parameters S11 and S22 refer to the RF signal reflection at port 1 and port 2, respectively, which were identical in the measurement. To achieve perfect isolation, the RF signal was totally 
a
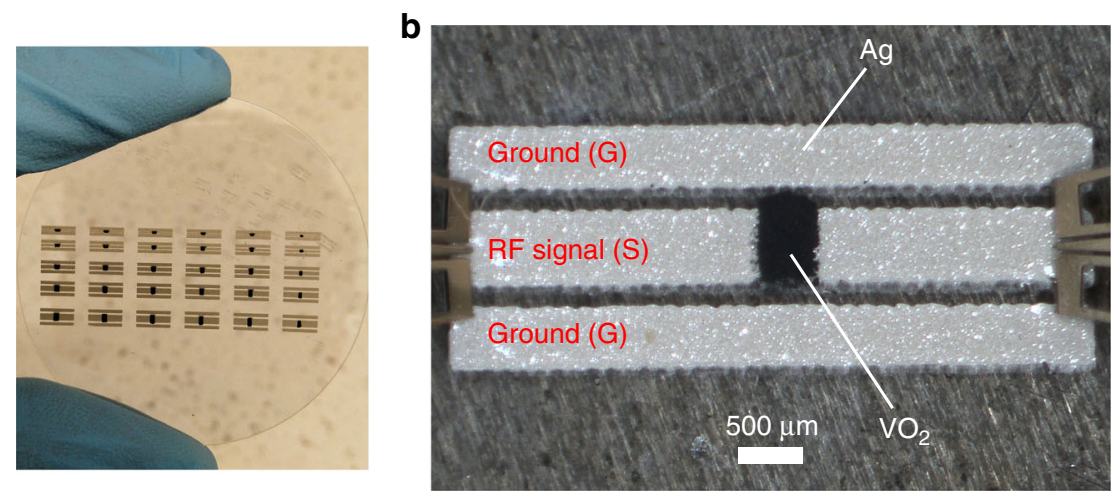

C

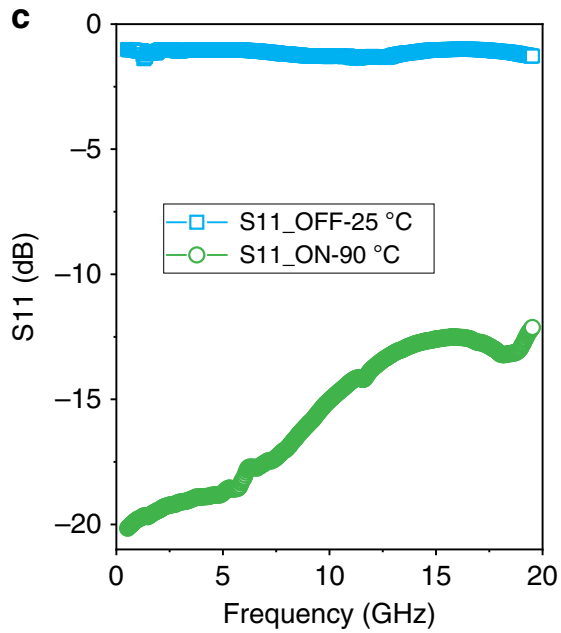

d

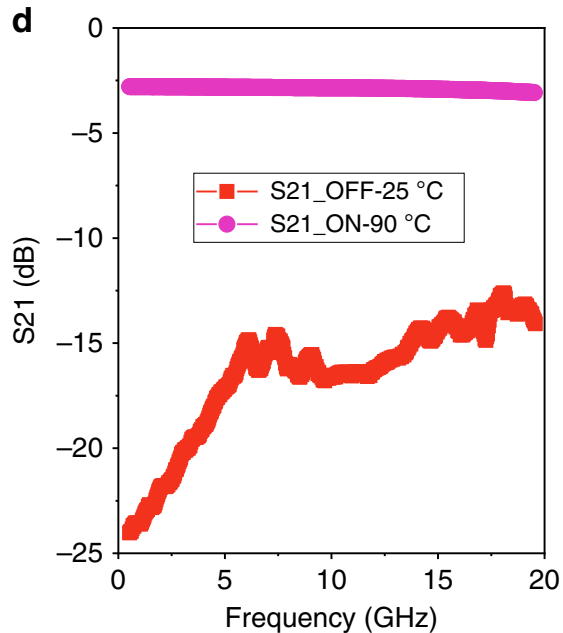

Fig. 5 The RF performance of the printed $\mathrm{VO}_{2}$ series switches. a Digital photograph of the fully screen-printed series switches on a sapphire wafer. b Optical image of one series switch under the RF performance test. $\mathbf{c}$ The measured $\mathrm{S} 11$ of the series switch at $24^{\circ} \mathrm{C}$ and $90^{\circ} \mathrm{C}$. $\mathbf{d}$ The measured S21 of the series switch at $24^{\circ} \mathrm{C}$ and $90^{\circ} \mathrm{C}$

reflected, and the measured S11 (or S22) was $0 \mathrm{~dB}$. Similarly, S12 and S21 indicated the RF signal transmission from port 1 to port 2 and from port 2 to port 1 , respectively, which were coincident with each other. For an ideal transmission, the RF signal is completely transmitted from one port to the other, and the measured S21 (or S12) should be $0 \mathrm{~dB}$. For our screen-printed series switches, at room temperature (OFF state), the measured S11 was approximately $-1 \mathrm{~dB}$ over the entire measured frequency range up to $20 \mathrm{GHz}$ (Fig. 5c), indicating satisfactory isolation. This was also confirmed by the S21 measurement, in which less than $-13 \mathrm{~dB}$ was observed. In contrast, S11 became lower than $-12 \mathrm{~dB}$, and $\mathrm{S} 21$ increased to $-2.6 \mathrm{~dB}$ (also called an insertion loss) when the temperature was $90^{\circ} \mathrm{C}$, demonstrating a reasonable transmission of the RF signal. These results indicated that our screen-printed $\mathrm{VO}_{2}$ switches demonstrated decent $\mathrm{RF}$ performance with an acceptable value of $-2.6 \mathrm{~dB}$ for an RF switch, though it is larger than a typical value of $-1.5-2.0 \mathrm{~dB}$ for a nonprinted $\mathrm{VO}_{2}$ switch through nanofabrication techniques ${ }^{29}$. To further identify this, a band-stop filter was fabricated on glass, and the RF performance was characterized. As illustrated in Fig. S9a in the Supporting Information, the filter was a microstripbased $\mathrm{T}$ resonator and contained three $\mathrm{VO}_{2}$ switches to adjust the length and width of the stub, enabling the tunability of both the resonant frequency and bandwidth. Here, two frequencies, $1.9 \mathrm{GHz}(\mathrm{GSM})$ and $2.4 \mathrm{GHz}$ (Bluetooth) were selected as a proof of concept, while any other frequency could be selected by simply changing the electrical length of the open stub in the T resonator. One prototype is presented in Fig. S9b that contained a fully printed filter and a printed heater element to activate the $\mathrm{VO}_{2}$ switches. The RF performance was measured using a vector network analyzer E8363C from Agilent. Fig. S9c-e presents the measured S-parameters (S11 and S21) of the filter under three different operating conditions in accordance with the three working conditions of $\mathrm{VO}_{2}$ switches. Table S1 in the Supporting Information summarizes the detailed measurement results. We observed well-matched results between the measurement and simulation. The resonant frequency of the filter shifted 


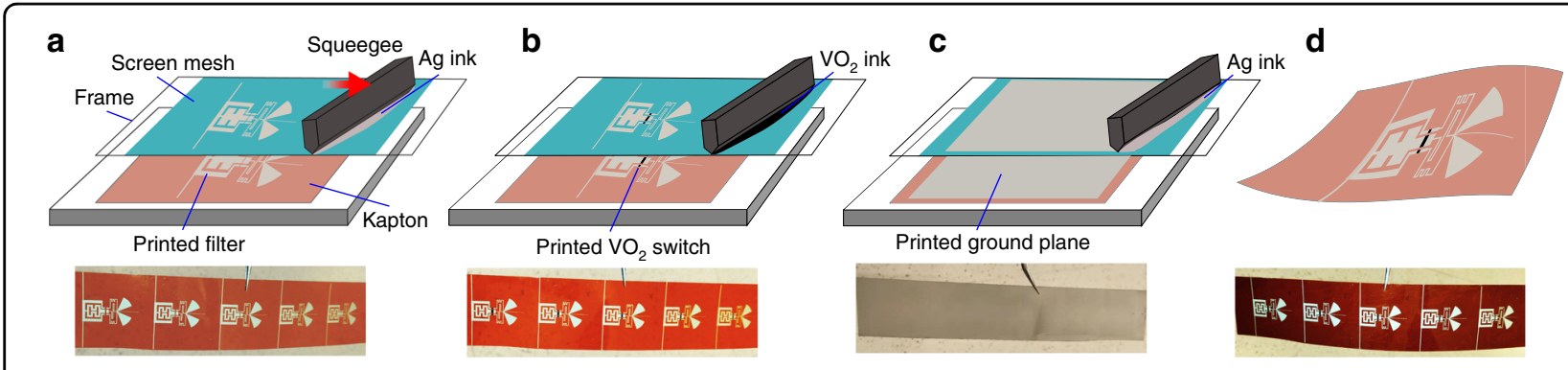

Fig. 6 Schematic illustration of fully screen-printed reconfigurable band stop filters. a Screen printing of the filters with Ag ink. b Screen printing of the $\mathrm{VO}_{2}$ switches with $\mathrm{VO}_{2}$ ink. c Screen printing of the ground plane with Ag ink. $\mathbf{d}$ The fabricated filter

from $2.4 \mathrm{GHz}$ to $1.9 \mathrm{GHz}$ when switch one was $\mathrm{ON}$. The bandwidth was considerably widened from 320 to $500 \mathrm{MHz}$ at $2.4 \mathrm{GHz}$ when switch two was $\mathrm{ON}$ and from 200 to $520 \mathrm{MHz}$ at $1.9 \mathrm{GHz}$ when all three switches were in the ON state. These results matched well with the simulation, providing confirmation of the reliable RF performance of our screen-printed $\mathrm{VO}_{2}$ switches.

The excellent electrical performance, together with decent RF performance, makes our screen-printable $\mathrm{VO}_{2}$ ink suitable for high-performance, tunable, and reconfigurable RF electronics. As a proof of concept, we demonstrated the high-throughput production of fully screen-printed and flexible band-pass filters on a Kapton film. Figure 6 illustrates the fabrication steps, which consist of (a) screen-printing of band-pass filters on the Kapton film; (b) screen-printing of $\mathrm{VO}_{2}$ switches in the desired areas; and (c) screen-printing of the ground plane. The printed samples in each step are also shown in the bottom panel of Fig. 6, in which six filters were produced in one cycle to improve the fabrication efficiency. For convenience, a common ground plane was printed for all the switches, as shown in Fig. 6c. However, for the measurements, each filter was cut separately to avoid any electrical connection. A photograph of the fabricated filters is displayed in Fig. 6d.

As presented in Fig. S10 in the Supporting Information, the designed filter contains three parts: a main body (marked as dark gray), $\mathrm{VO}_{2}$ switches (marked as red), and a triggering component (marked as light gray). The three switches were used to tune the length of the filter, allowing an RF signal pass through at a center frequency of $4 \mathrm{GHz}$ with a $500 \mathrm{MHz}$ bandwidth at the OFF state, while the working frequency shifted to $3.75 \mathrm{GHz}$ when the switch was $\mathrm{ON}$. The triggering lines were designed to inject bias current to activate the $\mathrm{VO}_{2}$ switches. The detailed geometrical dimensions of the designed filter and the 3D model in the full-wave simulator are depicted in Fig. S10. Furthermore, Fig. 7a presents one filter prototype ready to test, which was connected with two subminiature version A connectors to transmit an RF signal and a DC wire (color in purple) to apply current. The optical images of the printed $\mathrm{Ag}$ lines and $\mathrm{VO}_{2}$ switches are displayed in the right panel of Fig. 7a, demonstrating fairly clear and smooth edges that enable the stability and reproducibility of RF electronic devices. A diagram and digital photograph of the apparatus to measure the Sparameters of the fabricated filter is presented in Fig. $7 \mathrm{~b}$. Each end of the filter was connected to a bias Tee, which combined the RF signal from a precalibrated Vector Network Analyzer (VNA) with the DC current from a current source to provide an RF signal and bias current to the filter. The S-parameters were recorded by the VNA and replotted in Fig. $7 \mathrm{c}, \mathrm{d}$, together with the simulation. If no current was applied, the three $\mathrm{VO}_{2}$ switches were in the OFF state. In this case, the triggering component was disconnected from the main body of the filter, resulting in a shortened filter resonating at a high frequency, i.e. $4 \mathrm{GHz}$. The measured S21 exhibited a center frequency of $3.95 \mathrm{GHz}$ with an insertion loss of $4.3 \mathrm{~dB}$, while an operating frequency of $4 \mathrm{GHz}$ with an insertion loss of $3.9 \mathrm{~dB}$ was obtained from the simulation. The pass-band widths (frequencies at $3 \mathrm{~dB}$ attenuation) were 0.45 and $0.48 \mathrm{GHz}$ for the measurement and simulation, respectively. Similar results were also observed from the S11 curves, as presented in Fig. $7 d$. In contrast, the three $\mathrm{VO}_{2}$ switches were activated in the ON state when the DC current $(200 \mathrm{~mA})$ was injected into the filter through the triggering component. Correspondingly, the triggering component was connected to the filter body, which resulted in a long filter operating at a low frequency (i.e., $3.75 \mathrm{GHz}$ ). As presented in the S21 curves in Fig. 7c, the center frequency for the measurement and simulation were 3.77 and $3.72 \mathrm{GHz}$, respectively, while there was a $0.7 \mathrm{~dB}$ difference in the insertion loss. Furthermore, we observed that the passband widths were $0.42 \mathrm{GHz}$ and $0.31 \mathrm{GHz}$ for measurement and simulation, respectively. The resonant frequencies in the S11 curves demonstrated similar results. Although the measured RF performance of the fabricated filter matched well with the simulated results, minor shifts in the center frequency, mismatched 

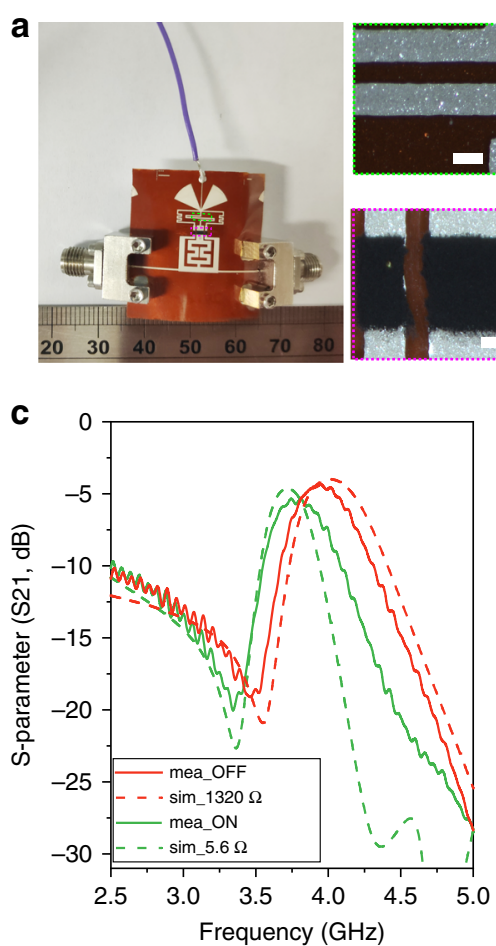
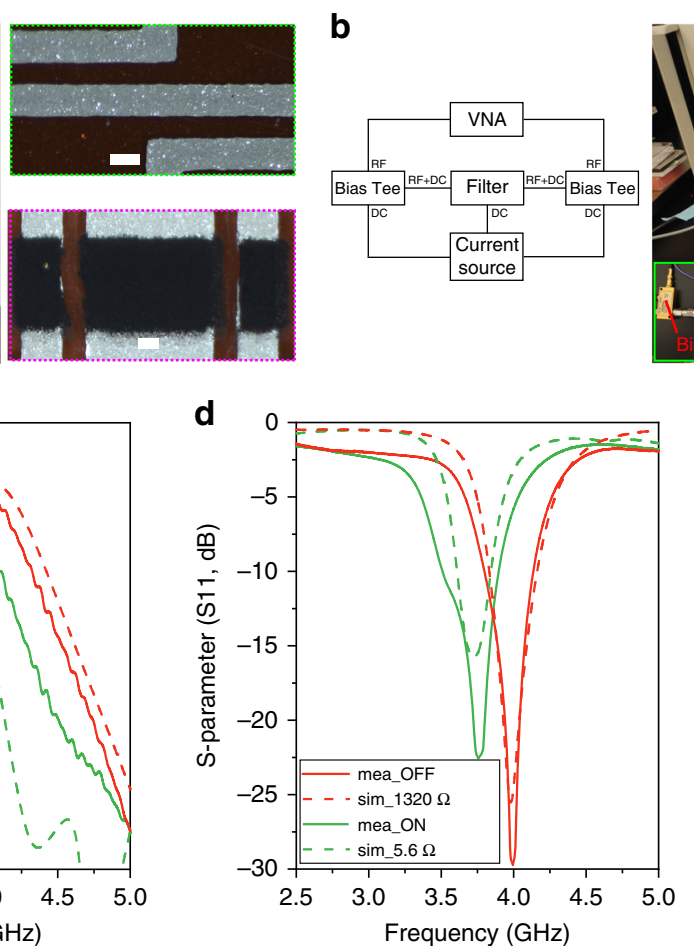
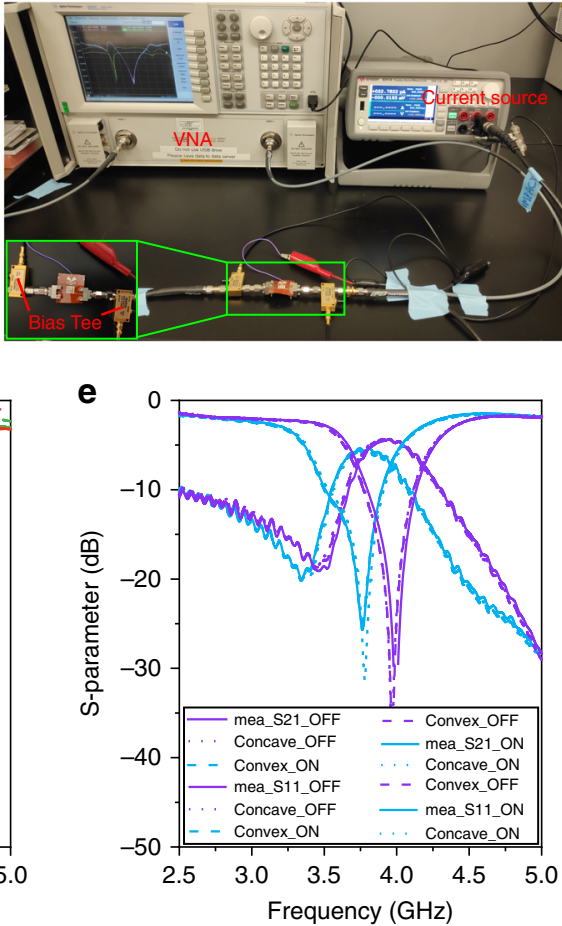

Fig. 7 Performance of the fully screen-printed, flexible band-pass filters. a Digital photograph of one filter (left panel) with stimulating DC line and RF connectors. Right panel: optical images of the printed Ag lines and $\mathrm{VO}_{2}$ switches. Scale bars are $200 \mu \mathrm{m}$. b Schematic illustration (left panel) and digital photograph of the measurement apparatus. c The measured and simulated S21 of the filter at the OFF and ON states. $\mathbf{d}$ The measured and simulated S11 of the filter at the OFF and ON states. e The measured S11 and S21 of the filter at the OFF and ON states under various bending conditions

bandwidth, and rippled curves were observed. This could be attributed to several reasons: (1) the printed Ag lines were slightly wider than the designed ones due to the ink spreading on the substrate, as confirmed by Fig. 2e; (2) the surfaces of the printed $\mathrm{Ag}$ lines and $\mathrm{VO}_{2}$ layers were not smooth (Fig. S11a, b), resulting in possible wave diffraction; (3) the polymer binders in the Ag and $\mathrm{VO}_{2}$ layers (Figs. S11c and S7) negatively affected the RF performance; and (4) the calibration tolerance. We then concluded by investigating the flexibility of the fabricated filter by measuring the S-parameters under bending conditions, i.e., convex and concave directions. Figure 7e displays the measured results of S11 and S21, in which we found well-matched curves for various bending conditions in both the OFF and ON states, demonstrating consistent performance during mechanical deformation and supporting potential applications in flexible RF electronic devices.

\section{Discussion}

In summary, a screen-printable $\mathrm{VO}_{2}$ ink was developed by dispersing high-purity $\mathrm{VO}_{2}$ microparticles into a viscous EC matrix. $\mathrm{VO}_{2}$ switches with uniform and clear edges were printed with the ink on flexible polymers and rigid wafers over a large area and with a high efficiency (up to $220 \mathrm{~mm} \mathrm{~s}^{-1}$ ). A low annealing temperature of $120^{\circ} \mathrm{C}$ was used to sinter the printed $\mathrm{VO}_{2}$ switches to achieve a low $\mathrm{ON}$ resistance (as low as $1.8 \mathrm{ohm}$ ), high electrical conductivity (larger than $1000 \mathrm{~S} \mathrm{~m}^{-1}$ ), and excellent ON/OFF ratio (more than 300). Both thermal and electrical triggers were successfully used as stimuli to trigger the $\mathrm{VO}_{2}$ switches. When integrated into $\mathrm{CPW}$ microstripe lines, the screen-printed $\mathrm{VO}_{2}$ switch controlled the transmission of the RF signal, demonstrating a $-1 \mathrm{~dB}$ isolation and $-2.6 \mathrm{~dB}$ transmission at $25^{\circ} \mathrm{C}$ (OFF state) and $90^{\circ} \mathrm{C}$ (ON state), respectively. The $\mathrm{VO}_{2}$ switches were successfully integrated into fully screenprinted frequency reconfigurable RF electronics (i.e., band-stop filter and band-pass filter). A batch of six bandpass filters was printed on Kapton film in one printing process. The center frequency of the filters was tuned from 3.95 to $3.77 \mathrm{GHz}$ after applying a DC current of $200 \mathrm{~mA}$ via the triggering component. Moreover, the measured S-parameters were consistent under flat and bending conditions, implying high mechanical stability. All these results present a promising approach for realizing reconfigurable RF electronics through a fully screen-printed process. 


\section{Materials and methods Chemicals}

Vanadium (V) oxide $\left(\mathrm{V}_{2} \mathrm{O}_{5}, \geq 98 \%\right.$, Sigma-Aldrich), oxalic acid dihydrate $\left(\mathrm{HO}_{2} \mathrm{CCO}_{2} \mathrm{H} \cdot 2 \mathrm{H}_{2} \mathrm{O}, \geq 99 \%\right.$, ACS reagent, Sigma-Aldrich), ethyl cellulose (EC, SigmaAldrich), terpineol (ACS reagent, Sigma-Aldrich), and ethanol (>99\%, Sigma-Aldrich) were used as received without further purification.

\section{Synthesis of $\mathrm{VO}_{2}$ microparticles}

In a typical synthesis process, $3 \mathrm{~g}$ oxalic acid was dissolved in $150 \mathrm{~mL}$ deionized (DI) water, and $0.5 \mathrm{~g}$ of vanadium $(\mathrm{V})$ oxide powder was subsequently added. The resultant mixture was mixed well until becoming a yellow-colored slurry solution. The final solution was then transferred into a $200 \mathrm{~mL}$ PPL high-temperature polymerliner-based hydrothermal autoclave reactor. The reaction temperature was set to $240{ }^{\circ} \mathrm{C}$. Three sets of experiments were performed, varying the reaction times from 3-24 h. After the completion of the reaction, the resultant blueblack precipitate was centrifuged, washed with water and ethanol and then dried in a vacuum furnace at $70{ }^{\circ} \mathrm{C}$ for $1 \mathrm{~h}$. The obtained dried powder was then annealed at $300{ }^{\circ} \mathrm{C}$ for $3 \mathrm{~h}$ in a vacuum furnace to obtain $\mathrm{VO}_{2}(\mathrm{M})$ microparticles.

\section{Ink formulation and screen printing}

First, a viscous organic binder solution was formulated by mixing EC, terpineol, and ethanol at a weight ratio of 1:4:0.4. Prior to printing, the obtained pure $\mathrm{VO}_{2}$ particles were mixed with the prepared solution at a weight ratio of $3: 5$, followed by agitation to obtain the $\mathrm{VO}_{2}$ ink. Screen printing was performed on a professional printing system (AUREL screen printer 900PA) with a 21 inch $\times 21$ inch stainless-steel screen mesh mask (325 mesh count, $22.5^{\circ}$ mesh angle, $10 \mu \mathrm{m}$ emulsion thickness) at a printing speed of $220 \mathrm{~mm} \mathrm{~s}^{-1}$. The $\mathrm{VO}_{2}$ switches were printed at the gaps between the silver traces, which were screen printed using commercial silver paste (PE819, DuPont) and annealed at $300{ }^{\circ} \mathrm{C}$ for $1 \mathrm{~h}$ to obtain a conductivity of $1.8 \times 10^{7} \mathrm{~S} \mathrm{~m}^{-1}$. Finally, the printed $\mathrm{VO}_{2}$ switches were sintered at $120^{\circ} \mathrm{C}$ for $1 \mathrm{~h}$.

\section{Filter simulation}

A professional simulator (CST Microwave Studio 2019) was used for the full-wave simulation, and the constructed 3D model of the filter is presented in Fig. S10. The silver traces were modeled as a sheet with a conductivity of $1.8 \times 10^{7} \mathrm{~S} \mathrm{~m}^{-1}$, a thickness of $8 \mu \mathrm{m}$, and a surface roughness of $1 \mu \mathrm{m}$. The dielectric substrate had a permittivity of $2.8 \pm 0.02 \mathrm{j}$ and a thickness of $125 \mu \mathrm{m}$. The $\mathrm{VO}_{2}$ switches were modeled as lumped elements with resistances of $5.6 \mathrm{ohms}$ and $1320 \mathrm{ohms}$. The two sources were molded as a discrete 50 -ohm port. The frequency range was set at $2.5-5 \mathrm{GHz}$.

\section{Characterization}

The morphologies of the $\mathrm{VO}_{2}$ particles were characterized by scanning electron microscopy (ZEISS Merlin) and transmission electron microscopy (FEI Titan G2). The structure of the $\mathrm{VO}_{2}$ particles was characterized by XRD (Bruker D2 PHASER). The DSC was performed using the Discovery DSC tool. The rheological behavior of the formulated $\mathrm{VO}_{2}$ ink was tested using an AR1500 rheometer (TA Instruments). The thickness of the $\mathrm{VO}_{2}$ film was measured using a surface profiler (Veeco Dektak 150). The RF measurements were performed using an Agilent N5225A Vector Network Analyzer. The Sparameters of the filters were acquired from 2.5 to $5 \mathrm{GHz}$ after RF calibration using the standard short-openload (SOL) method to eliminate parasitic effects of the measurement system.

\section{Acknowledgements}

W.L. and M.V. contributed equally to this work. Special thanks to KAUST for funding.

\section{Author contributions}

All authors contributed to the preparation of this paper. W.L. and M.V. conceived the idea. M.V. synthesized and characterized the materials. W.L. formulated the ink, fabricated the devices, performed the tests, and analyzed the data. S.Y. designed the filters and helped perform the test and analyzed the data. A.S. supervised the research and corrected the paper.

\section{Data availability}

The datasets generated during and/or analyzed during the current study are available from the corresponding author on reasonable request.

Conflict of interest

The authors declare that they have no conflict of interest.

Supplementary information accompanies this paper at https://doi.org/ 10.1038/s41378-020-00194-2.

Received: 31 March 2020 Revised: 7 June 2020 Accepted: 21 June 2020 Published online: 05 October 2020

\footnotetext{
References

1. Dubal, D. P., Chodankar, N. R., Kim, D.-H. \& Gomez-Romero, P. Towards flexible solid-state supercapacitors for smart and wearable electronics. Chem. Soc. Rev. 47, 2065-2129 (2018)

2. Li, L., Bai, Y., Li, L., Wang, S. \& Zhang, T. A superhydrophobic smart coating for flexible and wearable sensing electronics. Adv. Mater. 29, 1702517 (2017).

3. Huang, W.-R., He, Z., Wang, J.-L., Liu, J.-W. \& Yu, S.-H. Mass production of nanowire-nylon flexible transparent smart windows for PM2.5 capture. iscience 12, 333-341 (2019)

4. Lee, S. J. et al. VO2MO3-based hybrid smart windows with thermochromic and electrochromic properties. ACS Sustain. Chem. Eng. 7, 7111-7117 (2019).

5. Kim, Y., Yuk, H., Zhao, R., Chester, S. A. \& Zhao, X. Printing ferromagnetic domains for untethered fast-transforming soft materials. Nature $\mathbf{5 5 8}, \mathbf{2 7 4 - 2 7 9}$ (2018).

6. Wang, S. et al. Warm/cool-tone switchable thermochromic material for smart windows by orthogonally integrating properties of pillar[6]arene and ferrocene. Nat. Commun. 9, 1737 (2018).
} 
7. Someya, T. \& Amagai, M. Toward a new generation of smart skins. Nat. Biotechnol. 37, 382-388 (2019).

8. Lee, Y. et al. Mimicking human and biological skins for multifunctional skin electronics. Adv. Funct. Mater. 30, 1904523 (2020).

9. Stadlober, B., Zirkl, M. \& Irimia-Vladu, M. Route towards sustainable smart sensors: ferroelectric polyvinylidene fluoride-based materials and their integration in flexible electronics. Chem. Soc. Rev. 48, 1787-1825 (2019).

10. Song, Y. et al. High-efficiency self-charging smart bracelet for portable electronics. Nano Energy 55, 29-36 (2019).

11. Huang, G.-W., Xiao, H.-M. \& Fu, S.-Y. Wearable electronics of silver-nanowire/ Poly(dimethylsiloxane) nanocomposite for smart clothing. Sci. Rep. 5, 13971 (2015).

12. Yang, S., Vaseem, M. \& Shamim, A. Fully inkjet-printed VO2-based radio-frequency switches for flexible reconfigurable components. Adv. Mater. Technol. 4, 1800276 (2019).

13. Vaseem, M., Ghaffar, F. A., Farooqui, M. F. \& Shamim, A. Iron oxide nanoparticlebased magnetic ink development for fully printed tunable radio-frequency devices. Adv. Mater. Technol. 3, 1700242 (2018).

14. Li, W. et al. A temperature-activated nanocomposite metamaterial absorber with a wide tunability. Nano Res. 11, 3931-3942 (2018).

15. Pan, K. et al. Sustainable production of highly conductive multilayer graphene ink for wireless connectivity and loT applications. Nat. Commun. 9, 5197 (2018).

16. Liu, X. et al. Recent advances in covalent organic frameworks (COFs) as a smart sensing material. Chem. Soc. Rev. 48, 5266-5302 (2019).

17. Vaseem, M., Zhen, S., Yang, S., Li, W. \& Shamim, A. Development of VO2nanoparticle-based metal-insulator transition electronic ink. Adv. Electron. Mater. 5, 1800949 (2019).

18. Xue, F. et al. MoS2 tribotronic transistor for smart tactile switch. Adv. Funct. Mater. 26, 2104-2109 (2016).

19. Song, J. \& Zeng, H. Transparent electrodes printed with nanocrystal inks for flexible smart devices. Angew. Chem. Int. Ed. 54, 9760-9774 (2015).

20. Bragaglia, V. et al. Metal-insulator transition driven by vacancy ordering in GeSbTe phase change materials. Sci. Rep. 6, 23843 (2016).

21. Hollander, M. J. et al. Electrically driven reversible insulator-metal phase transition in 1T-TaS2. Nano Lett. 15, 1861-1866 (2015).

22. Butakov, N. A. et al. Switchable plasmonic-dielectric resonators with metal-insulator transitions. ACS Photonics 5, 371-377 (2018).

23. Li, D. et al. Joule heating-induced metal-insulator transition in epitaxial VO2/ TiO2 devices. ACS Appl. Mater. Interfaces 8, 12908-12914 (2016).

24. Zhang, Z. et al. Nanoscale engineering in VO2 nanowires via direct electron writing process. Nano Lett. 17, 851-855 (2017).

25. Li, M., Magdassi, S., Gao, Y. \& Long, Y. Hydrothermal synthesis of VO2 polymorphs: advantages, challenges and prospects for the application of energy efficient smart windows. Small 13, 1701147 (2017).

26. Long, S. et al. Application-oriented VO2 thermochromic coatings with composite structures: optimized optical performance and robust fatigue properties. Sol. Energy Mater. Sol. Cells 189, 138-148 (2019).

27. Hou, J., Wang, Z., Ding, Z., Zhang, Z. \& Zhang, J. Facile synthesize VO2 (M1) nanorods for a low-cost infrared photodetector application. Sol. Energy Mater. Sol. Cells 176, 142-149 (2018).

28. Guo, D. et al. Hydrothermal one-step synthesis of highly dispersed M-Phase VO2 nanocrystals and application to flexible thermochromic film. ACS Appl. Mater. Interfaces 10, 28627-28634 (2018).
29. Huitema, L., Crunteanu, A., Wong, H. \& Arnaud, E. Highly integrated VO2-based tunable antenna for millimeter-wave applications. Appl. Phys. Lett. 110, 203501 (2017).

30. Gagaoudakis, E. et al. Low temperature if-sputtered thermochromic VO2 films on flexible glass substrates. Adv. Mat. Lett. 8, 757-761 (2017).

31. Gagaoudakis, E. et al. Low-temperature if sputtered VO2 thin films as thermochromic coatings for smart glazing systems. Sol. Energy 165, 115-121 (2018).

32. Umar, Z. et al. Substrate temperature effects on the structural, compositional, and electrical properties of VO2 thin films deposited by pulsed laser deposition. Surf. Interface Anal. 50, 297-303 (2018).

33. Kim, S., Shamim, A., Georgiadis, A., Aubert, H. \& Tentzeris, M. M. Fabrication of fully inkjet-printed vias and SIW structures on thick polymer substrates. IEEE Trans. Compon. Packag. Manuf. Technol. 6, 486-496 (2016).

34. Su, Z., Klionovski, K., Bilal, R. M. \& Shamim, A. A dual band additively manufactured 3-D antenna on package with near-isotropic radiation pattern. IEEE Trans. Antennas Propag. 66, 3295-3305 (2018).

35. Farooqui, M. F., Claudel, C. \& Shamim, A. An inkjet-printed buoyant 3-D Lagrangian sensor for real-time flood monitoring. IEEE Trans. Antennas Propag. 62, 3354-3359 (2014).

36. Meredov, A., Klionovski, K. \& Shamim, A. Screen-printed, flexible, parasitic beam-switching millimeter-wave antenna array for wearable applications. IEEE Open J. Antennas Propag. 1, 2-10 (2020).

37. Haghzadeh, M. \& Akyurtlu, A. All-printed, flexible, reconfigurable frequency selective surfaces. J. Appl. Phys. 120, 184901 (2016).

38. Quddious, A. et al. Disposable, paper-based, inkjet-printed humidity and H2S gas sensor for passive sensing applications. Sensors 16, 2073 (2016).

39. Ji, H., Liu, D., Cheng, H. \& Tao, Y. Large area infrared thermochromic VO2 nanoparticle films prepared by inkjet printing technology. Sol. Energy Mater. Sol. Cells 194, 235-243 (2019).

40. Li, W. et al. Synthesis of VO2 nanoparticles by a hydrothermal-assisted homogeneous precipitation approach for thermochromic applications. RSC Adv. 4, 13026-13033 (2014).

41. $\mathrm{Xu}, \mathrm{H}$. et al. Vertically distributed VO2 nanoplatelets on hollow spheres with enhanced thermochromic properties. J. Mater. Chem. C. 6, 7896-7904 (2018).

42. Liang, S., Shi, Q., Zhu, H., Peng, B. \& Huang, W. One-step hydrothermal synthesis of W-doped VO2 (M) nanorods with a tunable phase-transition temperature for infrared smart windows. ACS Omega 1, 1139-1148 (2016).

43. Li, X. et al. A convenient way to reduce the hysteresis width of $\mathrm{VO} 2(\mathrm{M})$ nanomaterials. N. J. Chem. 41, 15260-15267 (2017).

44. Wu, C., Feng, F. \& Xie, Y. Design of vanadium oxide structures with controllable electrical properties for energy applications. Chem. Soc. Rev. 42, 5157-5183 (2013).

45. Liu, M., Su, B., Tang, Y., Jiang, X. \& Yu, A. Recent advances in nanostructured vanadium oxides and composites for energy conversion. Adv. Energy Mater. 7, 1700885 (2017).

46. Shao, Z., Cao, X., Luo, H. \& Jin, P. Recent progress in the phase-transition mechanism and modulation of vanadium dioxide materials. NPG Asia Mater. 10, 581-605 (2018).

47. Potts, S.-J. et al. High-speed imaging the effect of snap-off distance and squeegee speed on the ink transfer mechanism of screen-printed carbon pastes. J. Coat. Technol. Res. 17, 447-459 (2020).

48. PAN, J., TONKAY, G. L. \& QUINTERO, A. Screen printing process design of experiments for fine line printing of thick film ceramic substrates. J. Electron. Manuf. 09, 203-213 (1999). 